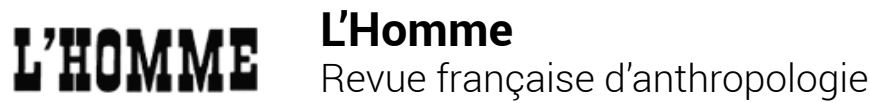

187-188 | 2008

Miroirs transatlantiques

\section{Morgan Jouvenet, Rap, techno, électro... Le musicien entre travail artistique et critique sociale}

\section{Monique Jeudy-Ballini}

\section{(2) OpenEdition}

1 Journals

\section{Édition électronique}

URL : https://journals.openedition.org/lhomme/20972

DOI : 10.4000/lhomme.20972

ISSN : 1953-8103

Éditeur

Éditions de l'EHESS

\section{Édition imprimée}

Date de publication : 3 octobre 2008

Pagination : 525-527

ISBN : 978-2-7132-2186-6

ISSN : 0439-4216

\section{Référence électronique}

Monique Jeudy-Ballini, « Morgan Jouvenet, Rap, techno, électro... Le musicien entre travail artistique et critique sociale », L'Homme [En ligne], 187-188 | 2008, mis en ligne le 16 décembre 2008, consulté le 23 avril 2022. URL : http://journals.openedition.org//homme/20972 ; DOI : https://doi.org/10.4000/ Ihomme.20972

Ce document a été généré automatiquement le 23 avril 2022.

(c) École des hautes études en sciences sociales 


\title{
Morgan Jouvenet, Rap, techno, électro... Le musicien entre travail artistique et critique sociale
}

\author{
Monique Jeudy-Ballini
}

\section{RÉFÉRENCE}

Morgan JOUVENET, Rap, techno, électro... Le musicien entre travail artistique et critique sociale. Paris, Éd. de la Maison des sciences de l'homme, 2006, 290 p., bibl. (« Ethnologie de la France »).

1 RAP, TECHNO, ÉLECTRO : ces genres qui se mettent à bousculer l'industrie musicale dans les années 1980, ces genres que leur diversité stylistique, leur plasticité, leur esthétique déconstructionniste et la courte temporalité de leurs productions tendent à rendre insaisissables, comment en fixer l'histoire? «Plus cette histoire devient précise, plus c'est l'éclatement du fait musical qui s'impose et son objet qui devient flou ", écrit Morgan Jouvenet (pp.14-15). En commençant par examiner leur genèse, il met en évidence le lien consubstantiel de ces musiques avec les environnements sociaux dont elles sont issues et dont elles continuent de se nourrir.

2 L'immigration, le vécu urbain difficile, la rébellion, le mélange des genres..., c'est notamment cela qu'il s'agit pour elles de bricoler en un recyclage continu et parfois improvisé de matière sonore. Récupération, collage, détournement, mais aussi abolition de la distance entre compositeurs et interprètes, auditeurs et musiciens, stars et inconnus: la réappropriation inventive passe par une volonté critique de décloisonnement qui met à son service des innovations techniques pour subvertir la relation traditionnelle à la création et faire œuvre d'insoumission face aux logiques marchandes dominantes. Les pressions, voire les stratégies d'intimidation physique exercées par les rappeurs auprès des médias ressortissent à ce parti pris de résistance. Clairement, la performance musicale - souvent comparée à un sport de combat - est 
une arme. Contre le marché du disque qu'elle cherche à « dynamiter de l'intérieur » (p. 87), mais également contre les pairs puisqu'elle n'est estimée efficace que si elle " "tue" tout le monde, "atomise la concurrence", la "déchire", réalise un "carnage", etc. C'est dans le rap que l'on a commencé à décrire une bonne chanson comme une "tuerie" ", apprend-on (p. 75). Et l'on pense irrésistiblement alors à ce que l'on sait de l'impact esthétique dans certaines cultures de Nouvelle-Guinée, lui aussi conçu comme une violence, une agression qui «tue " le spectateur, un rapport de force dont il doit idéalement sortir vaincu si la prestation dansée ou chantée est probante.

En prenant toutefois le parti de placer son étude à distance de l'image emblématique spectaculaire que ces formes musicales se complaisent à donner d'elles-mêmes - «la "rage" des rappeurs, la "folie" des raveurs " (p. 265) -, Morgan Jouvenet entend surtout analyser l'imbrication des liens qui, dans l'espace de travail artistique, associent la conception des œuvres, leur exécution et leur mode de diffusion. Il y excelle avec une particulière acuité anthropologique en explorant tout autant les représentations dont se soutient la pratique de ces types de musiques que la manière singulière dont leurs auteurs s'impliquent avec un esprit entrepreneurial décomplexé dans l'organisation professionnelle. «Les activités managériales ne sont plus perçues comme des activités parasitant l'expressivité ou le style mais comme des activités permettant aux œuvres d'agir plus efficacement dans le monde social. On peut donc dire que la tension entre l'art et le management (ou le commerce) est comme prise en charge par ces cultures musicales ; elle n'est plus problématique en tant que telle pour la majorité des rappeurs et des électronistes ", contrairement, observe-t-il, à ce qu'elle fut un temps pour les jazzmen (p. 122).

4 Au fil de l'analyse, c'est toujours, fondamentalement, de la dialectique des rapports entre groupe et individu qu'il est question: d'un côté l'ancrage des artistes dans des contextes sociaux spécifiques, leur appartenance à une communauté de pairs, la nature résolument collective de leur création, l'adhésion à des conventions expressives partagées; mais de l'autre côté, et dans le même temps, des prises de parole à la première personne, des défis, un désir obsessionnel de se différencier, la revendication d'une identité irréductible. Pour le musicien, montre Morgan Jouvenet, le travail de positionnement « dans (et par) la maison de disques ", vise précisément à concilier ces tendances antagonistes, "à dépasser l'apparente contradiction entre les exigences d'originalité et de prévisibilité »(p. 181). C'est sur ce même registre ambivalent de l'inédit et du familier que s'appuient les récits du choc fondateur auquel les artistes font remonter leur parcours de musiciens, sorte de first contact avec le rap ou la techno qui, au détour d'un titre entendu à la radio ou d'une première sortie en rave party, dévoile accidentellement "un nouvel espace de vie, des perspectives d'action, d'émotion et de réflexion inédites » (p. 197). «L'insistance des artistes sur le "big bang biographique" initial, précise l'auteur, ne doit pas cependant nourrir l'illusion d'une rupture radicale dans les trajectoires individuelles [...] leurs évocations sont en effet aussi celles d'individus tout surpris de ne pas être surpris, reconnaissant quasi instantanément leur nouvel univers de référence comme étant "fait pour eux" ( $\mathrm{p}$. 199).

5 Entre rupture et continuité, changement («conversion») et fidélité à soi-même, l'itinéraire personnel conjugue indissociablement réflexion sur soi et réaction au monde. Mais «l'attention au monde n'a ici de sens que dans la perspective de l'invention de soi» (p. 257). L'authenticité constitue en la matière une valeur 
omniprésente. Le chapitre final que Jouvenet lui consacre permet, en une vue synthétique des différents aspects déjà examinés, d'en saisir le caractère puissamment organisateur au sein de ces univers musicaux où, tout à la fois, elle tient lieu de principe esthétique, et structure les relations professionnelles. Dans la conception de l'œuvre, l'authenticité, liée au fait d'avoir socialement souffert, s'actualise comme la sincérité émotionnelle, la spontanéité, l'expression directe, la veine réaliste, l'énergie critique et la violence libératoire qui érigent l'action musicale en vecteur de contrepouvoir (politique, éducatif, culturel...). À cela, un préalable : «ne pas (se) trahir » (p. 256), « accepter d'être durablement saisi par son origine sociale» (p. 270), ne cesser de dire d'où l'on vient et d'assigner à ses propositions artistiques une dimension édifiante subversive ; quel que soit le succès obtenu, consentir donc, en particulier, à ne jamais être quitte de sa dette envers le milieu marginal initial. «Tu peux sortir de la jungle/ cité mais tu ne peux pas sortir la jungle/cité qui est en toi », rappelle un slogan récurrent (p. 108). C'est que «la connaissance de soi est aussi [...] reconnaissance du collectif en soi. Même le plus grand artiste est en effet considéré comme le maillon d'une chaîne (de relations) sociale » (p. 270) ; idéologie fascinante que celle de cette filiation conceptuelle vue par les membres d'une communauté qui ne cessent pourtant de mettre en avant la performance individualisante et travaillent ensemble à la rendre insubstituable à toute autre.

Du remarquable ouvrage de Morgan Jouvenet, on retient la finesse sociologique d'une analyse qui, loin de toute dichotomie réductrice, dépasse constamment les paradoxes apparents pour penser les ambivalences. Sa capacité à rendre compte de la complexité d'un univers artistique en le contextualisant historiquement et en l'appréhendant à travers l'intrication de ses diverses composantes culturelles et professionnelles en fait une étude exemplaire ; une contribution aussi, en son genre, à l'anthropologie de l'art qui devrait y (re)trouver maints sujets de réflexion, à commencer par le thème général du livre sur le lien entre la nature des œuvres et la transformation des façons de travailler. Un autre concerne la place que ces esthétiques musicales portées par une critique de la rationalité occidentale accordent aux affects. L'émotion, cependant, y est moins l'expression d'un ressenti que la mise en scène de ce ressenti. L'art de dissimuler l'art consiste en l'occurrence à faire oublier aux auditeurs le professionnalisme des interprètes, à leur communiquer l'impression d'un refus de tricher, d'une véritable «transparence du vécu » selon des modes spécifiques d'expressivité devant permettre d'« entendre les coutures ", d'élaborer " une image sonore des processus de fabrication de la musique "en train de se faire" » (p. 119). Ainsi l'œuvre donne-t-elle à percevoir les coulisses de son élaboration, soit tout ce qu'il aurait été académiquement recommandé de gommer : ces ratages, débordements et autres imprévus passant pour authentifier l'« exposition volontaire à la contingence » (p. 96). Renvoyant à cette même esthétique de la vérité et au mépris affiché de tout carriérisme, « les photos mal cadrées ou à la mise au point défectueuse font partie de la panoplie promotionnelle de bien des musiciens soucieux de ne pas se "couler dans le moule" ni de devenir prisonniers d'une image arrêtée » (p. 88). La multiplication stratégique des pseudonymes publics pour un même interprète vise de la même façon à "conjurer l'emprisonnement identitaire inhérent à l'exploitation commerciale des disques" (p. 88). Dans la société contemporaine où la signature d'un artiste, à l'instar de toute marque de fabrique, est parfois si décisive qu'elle suffit à faire (l')œuvre, ce type de brouillage délibéré constitue un sujet de réflexion supplémentaire sur les nouvelles modalités de la réception de l'art. C'est également cette thématique des effets transformateurs induits 
par une pratique artistique qu'invite à considérer ce qui est dit du rap et de la redécouverte de l'écriture hors du cadre scolaire : « le projet global du rap est présenté comme concurrent des programmes éducatifs officiels, et les démonstrations par les rappeurs de leur goût pour le maniement de la langue ou de leur soif de connaissance sont interprétées comme des preuves de l'efficacité du premier par rapport aux seconds [...]. Imothep [un DJ] avance ainsi [...] que "les ventes des dictionnaires de synonymes ont triplé ces dernières années" et en conclut abruptement que "ce que l'Éducation nationale n'a pas réussi à faire, le rap l'a fait" » (p. 103). Si cette déclaration réjouissante renseigne certes surtout sur l'idée que ce locuteur a de l'impact de son art, il reste que celui-ci exprime un point de vue sur le monde social en cohérence indiscutable avec l'accessibilité à la fois matérielle et contre-institutionnelle des musiques rap et électroniques.

Il faudrait mentionner évidemment bien d'autres sujets de réflexion abordés dans cette lumineuse étude, notamment sur les rapports entre héritage et réappropriation esthétiques, palimpseste et création, logique artistique et logique industrielle, art et changement socioéconomique, ou encore sur la dimension humoristique présente dans les textes de rap par exemple. Ce livre riche et porté par une connaissance impressionnante du terrain d'enquête est en outre émaillé de citations saisissantes qui lui donnent sa chair et son tour alerte. Gageons que beaucoup de ceux qui le liront aimeraient l'avoir écrit. 